\title{
Proposed diagnostic criteria and the screening and diagnosing tool of Internet addiction in college students
}

\author{
Chih-Hung Ko ${ }^{\mathrm{a}, \mathrm{b}}, \mathrm{Ju}-\mathrm{Yu} \mathrm{Yen}^{\mathrm{a}, \mathrm{c}}$, Sue-Huei Chen ${ }^{\mathrm{d}}$, Ming-Jen Yang ${ }^{\mathrm{a}, \mathrm{e}}$, \\ Huang-Chi Lin ${ }^{\mathrm{a}}$, Cheng-Fang Yen ${ }^{\mathrm{a}, \mathrm{f}, *}$ \\ ${ }^{a}$ Department of Psychiatry, Kaohsiung Medical University Hospital, Kaohsiung 807, Taiwan \\ ${ }^{\mathrm{b}}$ Graduate Institute of Medicine, College of Medicine, Kaohsiung Medical University, Kaohsiung 807, Taiwan \\ ${ }^{\mathrm{c}}$ Department of Psychiatry, Kaohsiung Municipal Hsiao-Kang Hospital, Kaohsiung Medical University, Kaohsiung 812, Taiwan \\ ${ }^{\mathrm{d}}$ Department of Psychology, National Taiwan University, Taipei 106, Taiwan \\ ${ }^{\mathrm{e}}$ Graduate Institute of Behavioral Science, Kaohsiung Medical University, Kaohsiung 807, Taiwan \\ ${ }^{\mathrm{f}}$ Department of Psychiatry, Faculty of Medicine, College of Medicine, Kaohsiung Medical University, Kaohsiung 807, Taiwan
}

\begin{abstract}
The aims of this study were to develop diagnostic criteria of Internet addiction for college students (DC-IA-C) with diagnostic interviews and to establish the optimal cutoff points of the Chen Internet Addiction Scale (CIAS) for the purposes of screening for and making the diagnosis of Internet addiction. A total of 216 college students (132 male and 84 female) were recruited in the study. The results demonstrated that 5/6 cutoff points of criteria A in DC-IA-C had the best diagnostic accuracy (95.9\%). Besides, 63/64 and 67/68 cutoff points of the CIAS were evaluated to be the best screening and diagnostic cutoff points, respectively. The DC-IA-C can provide health care professionals with a means to diagnose and communicate about Internet addiction among college students, and the screening and diagnostic cutoff points of CIAS could provide a screening instrument or a discriminative instrument in surveys for Internet addiction among college students.
\end{abstract}

(C) 2009 Elsevier Inc. All rights reserved.

\section{Introduction}

Internet use has become one of the most popular recreational and academic activities for college students because of its convenience and availability in universities. In universities in Taiwan, free unlimited Internet service is usually provided to freshman college students (who are living apart from their families for the first time). Previous reports demonstrate that $8 \%$ to $13 \%$ of undergraduates are addicted to the Internet, which has impaired individual psychological well-being, peer and family interactions, and academic performance [1-3]. Thus, Internet addiction has become an important issue of mental health for college students. Besides, college students have been one of the most mentioned target groups as well as adolescents and online users in previous reports for Internet addiction [4]. Because

\footnotetext{
* Corresponding author. Department of Psychiatry, Kaohsiung Medical University Hospital, Kaohsiung City 807, Taiwan. Tel.: +886 7 3121101x6822; fax: +88673134761 .

E-mail address: chfaye@cc.kmu.edu.tw (C.-F. Yen).
}

college students have different developmental tasks from adolescents, development of diagnostic criteria to define functional impairment specifically for college students is a necessity. However, until now, no diagnostic tool with welldeveloped sensitivity and specificity to detect those college students with Internet addiction has been developed.

The newly behavioral problems manifesting from heavy Internet use have been termed "Internet addiction" [4], "problematic Internet use" [5], or "pathological Internet use" [3]. Internet addition is the most widely used term to describe this maladaptive Internet use. Addiction has been previously defined to involve only substance use. However, Holden [6] had suggested that out-of-control gambling, eating, sexuality, and Internet use may share the same neurobiological mechanism with substance dependence and can be termed "behavioral addiction." Besides, Martin and Petry [7] also argued that addiction should include these uncontrolled behaviors. Thus, we have termed this uncontrolled Internet use as Internet addiction to represent its addictive characteristics.

Until now, there is no conclusive definition for Internet addiction. Anderson [8] has used the 7 true-false questions 
that most closely parallel the diagnostic criteria of substance dependence in Diagnostic and Statistical Manual of Mental Disorders, Fourth Edition (DSM-IV) [9] to define Internet dependence. Young [3] modified the diagnostic criteria of pathological gambling in DSM-IV to construct diagnostic criteria for pathological Internet use. Of those, she has defined 5 or more of 8 characteristic symptoms as cases of pathological Internet use. Shapira et al [10] defined problematic Internet use as uncontrollable; markedly distressing; time consuming or resulting in social, occupational, or financial difficulties; and not solely present during hypomanic and manic symptoms. In his study, all 20 subjects fitting the above definition also fit the DSM-IV criteria for impulse control disorder not elsewhere classified, based on the Structure Clinical Interview for DSM-IV Patient version. Then, Shapira et al [5] conceptualized Internet addiction as an impulse control disorder and proposed the diagnostic criteria of problematic Internet use be based on the general style of impulse control disorder in DSM-IV Text Revision (DSM-IV-TR) [11]. He suggests that one criterion of preoccupation of Internet use or excessive use of Internet longer than planned should exist to fulfill criteria A, and functional impairment and excluding criteria as defined in criteria B and C [5]. However, no control group is included to reveal the diagnostic potential of the criteria in these 2 reports. Aboujaoude et al [12] developed 4 sets of diagnostic criteria, which were created from diagnostic criteria of other impulse control disorders, obsessive-compulsive disorder, and substance abuse and from suggested criteria for problematic Internet use. The first set of criteria required regular Internet use, interference with relationships, preoccupation, and unsuccessful cutting down or staying online longer than intended to make diagnosis. All diagnostic criteria mentioned above have all tried to develop the concept for this newly developed disorder. However, the discriminative potential of selected criteria items and the sensitivity or specificity of these diagnostic criteria have not been provided.

Ko et al [13] have proposed diagnostic criteria of Internet addiction in adolescents (DC-IA-A) based on systemic diagnostic interview. In that study, Internet-addicted individuals and non-Internet-addicted individuals were classified based on both the global clinical impression of diagnostic interview and score on Chen Internet Addiction Scale (CIAS; higher than 75th percentile). A total of 13 candidate diagnostic criteria derived from diagnostic criteria of impulse control disorder and substance use disorder in DSM-IV-TR, plus diagnostic criteria for Internet addiction in previous studies and clinical experiences were selected to test their diagnostic accuracy in 486 adolescents recruited from junior high school. Three criteria were excluded due to lower diagnostic accuracy $(<75 \%)$. The criteria for functional impairment were selected as criteria B. Then, the best cutoff point with 6 of the remaining 9 criteria list in criteria $A$ was determined according to diagnostic accuracy. It provided a good diagnostic accuracy (95.4\%), with high specificity
(97.1\%) and accepted sensitivity (87.5\%) for adolescent Internet addiction. It has been reported to identify heavy $(>20$ hours per week) and frequent (everyday use) Internet users [13]. However, the validity of the DC-IA-A has not yet been examined in college students. Because the characteristics of Internet use might differ between adolescent and college student populations, establishing diagnostic criteria of Internet addiction for college students (DC-IA-C) based on empirical studies is critically necessary for the purposes of diagnosis and intervention in clinical settings.

To evaluate Internet addiction of college students, it would be optimal to conduct a 1-stage investigation by a brief self-reported instrument with high diagnostic accuracy. Besides, conducting a 2-stage diagnostic process in which a brief self-reported instrument is administered with a high sensitivity is the first step, followed by a diagnostic interview for screening positive users [14]. The CIAS is a 26-item selfreported measure for Internet addiction with good reliability and validity for college students [15]. The diagnostic (63/64) and screen cutoff point (57/58) of CIAS has been developed among adolescents with good sensitivity and specificity [16]. It has been used to evaluate personality and family function of Internet addiction among adolescents [17,18]. The evaluation of Internet addiction with a self-reported instrument in an epidemiology study is also necessary for college students. Thus, the development of a diagnostic or screening instrument with clear sensitivity and specificity is essential to do a sizeable and economical survey. However, no diagnostic or screening cutoff point for CIAS has been developed for college students.

Accordingly, the aims of this study were to develop DCIA-C with diagnostic interviews and to establish the optimal cutoff points of the CIAS for the purposes of screening for and making the diagnosis of Internet addiction among college students according to the DC-IA-C.

\section{Methods}

\subsection{Participants and procedure}

A total of 216 college students (132 men and 84 women) were invited to participate in this study. A total of 139 and 77 participants responded to the advertisement post for high (heavy use nearly every day) and low $(<2 \mathrm{~h} / \mathrm{d})$ levels of Internet use, respectively. Their mean age was $21.45 \pm 2.05$ years old (range, 18-27 years), and their average duration of education was $14.6 \pm 1.4$ years (range, $13-18$ years). There were 130,60 , and 26 participants recruited from medical, science and technological and general universities, respectively. All participants completed the CIAS, and the questionnaire assessing the frequency of Internet use and time spent online every week after informed consent was obtained. Then, 1 psychiatrist who was blinded to the result of the CIAS conducted diagnostic interviews for all participants based on the structured interview schedule of the DC-IA-C. The existence of every criterion was determined by any 
positive answer for questions evaluated for these criteria. Besides, the existence of Internet addiction was determined by the psychiatrist based on clinical impression.

\subsection{Instruments}

For diagnosing Internet addiction in college students, we developed the DC-IA-C, which was modified from the DCIA-A [13]. The DC-IA-A consists of 3 main sections, that is, 9 characteristic symptoms of Internet addiction (criteria A), functional impairment secondary to Internet use (criteria B), and the exclusive criteria (criteria C). In DC-IA-C, the criteria for functional impairment was modified to describe the negative impact of Internet addiction for college students. The first and secondary items of criteria B were modified to "recurrent Internet use resulting in a failure to fulfill major role obligations at work, school, and home" and "important social or recreational activities are given up or reduced because of Internet use" (Table 1). Besides, the object of other behavioral addictions, for example, pathological gambling, might exist online and represent maladaptive Internet use. Thus, other behavioral addictions classified in impulse control disorder or paraphilia in DSM-IV-TR [11] should be listed in criteria $\mathrm{C}$ to make further differential diagnosis.

Table 1

Proposed DC-IA-C

Distinguishing characteristics of Internet addiction

A maladaptive pattern of Internet use, leading to clinically significant impairment or distress, occurring at any time within the same 3-month period

A. Six (or more) of the following symptoms have been present

1. Preoccupation with Internet activities.

2. Recurrent failure to resist the impulse to use the Internet.

3. Tolerance: a marked increase in the duration of Internet use needed to achieve satisfaction.

4. Withdrawal, as manifested by either of the following

i. Symptoms of dysphoric mood, anxiety, irritability, and boredom after several days without Internet activity.

ii. Use of Internet to relieve or avoid withdrawal symptoms.

5. Use of Internet for a period longer than intended.

6. Persistent desire and/or unsuccessful attempts to cut down or reduce Internet use.

7. Excessive time spent on Internet activities and leaving the Internet.

8. Excessive effort spent on activities necessary to obtain access to the Internet.

9. Continued heavy Internet use despite knowledge of having a persistent or recurrent physical or psychological problem likely to have been caused or exacerbated by Internet use.

B. Functional impairment: one (or more) of the following symptoms have been present

1. Recurrent Internet use resulting in a failure to fulfill major role obligations at work, school, and home.

2. Important social or recreational activities are given up or reduced because of Internet use.

3. Recurrent legal problems because of Internet behavior. (eg, arrest for disorderly conduct in a game).

C. The Internet addictive behavior is not better accounted for by psychotic disorder, bipolar I disorder, or other disorder, which is classified in impulse control disorder and paraphilia in $D S M-I V-T R$.
Besides, as for substance dependence, the items of criteria A defined the symptoms in specific situations, for example, withdrawal or difficulty in cutting down. Thus, they might not experience them at most times. Thus, at least 1 period was necessary to collect the core symptoms. A 12-month period was defined for substance dependence. However, it has been reported that the 1-year remission rate of Internet addiction was $49.4 \%$ in adolescents [19]. Too long a duration, for example, 1 year, in collecting the symptomatic data may include symptoms in different episodes. On the other hand, college students with Internet addiction may fail to control Internet use after cutting-down for a short time. Too short a time in collecting symptoms may define a short withdrawal period as a remission state. Thus, based on our clinical experience, we suggest that 3 months may be an optimal duration for collecting these core symptoms.

The CIAS is a 4-point, 26-item self-reported scale assessing 5 dimensions of symptoms of compulsive use, withdrawal, tolerance, and problems of interpersonal relationships and health/time management [18]. The total scores of the CIAS ranged from 26 to 104 . Higher CIAS scores indicated increased severity of Internet addiction. The internal reliability of the scale and the subscales in the original study ranged from 0.79 to 0.93 , and correlation analyses yielded significantly positive correlation of total scale and subscale scores of CIAS with the hours spent weekly on Internet activity [18].

\subsection{Statistical analysis}

Sensitivity (true positive/true positive and false negative), specificity (true negative/true negative and false positive), diagnostic accuracy (true positive and true negative/all), positive predictive rate (PPR; true positive/true positive and false positive), negative predictive rate (NPR; true negative/ true negative and false negative), Cohen $\kappa$ value [20], and diagnostic odds ratio (DOR) [21] were all used to examine the diagnostic efficacy of cutoff points of criteria $\mathrm{A}$ in the DC-IA-C based on the diagnosis of clinical impression. The Cohen $\kappa$ value was used to compare the proportion of correct test-based classification to the proportion of correct classifications that would be expected with random assignment of diagnoses [22]. $\kappa$ values indicated if the agreement between measurements was poor $(<0.20)$, fair $(0.21-0.40)$, moderate $(0.40-0.60)$, good $(0.61-0.80)$, or very good $(0.81$ 1.00) [23]. The DOR of the cutoff point was calculated as the likelihood ratio for a positive result divided by the likelihood ratio for a negative result. Higher values of DOR indicated better discriminatory test performance, which did not depend on the prevalence of the target disease [21].

After the cutoff point of the DC-IA-C was validated, 2 cutoff points of the CIAS based on DC-IA-C were evaluated with the same indicators. One cutoff point was optimal for the diagnostic purpose in the 1-stage investigation if it resulted in a high diagnostic accuracy, Cohen $\kappa$ value, and DOR. The other cutoff point was optimal for screening the cases with Internet addiction in 
the 2-stage diagnostic process if it resulted in a high sensitivity and specificity higher than 75\% [24]. Besides, the area under the curve of receiver operating characteristics analysis was used to measure the diagnostic efficacy of the CIAS [25].

To confirm the validity of the cutoff points of DC-IA-C proposed in this study, the participants were further divided into the Internet addiction group and non-Internet addiction group according to DC-IA-C. The demographic data and characteristics of Internet use behaviors were further compared between the 2 groups by $\chi^{2}$ test. A $P$ value less than .05 was considered statistically significant.

\section{Results}

After diagnostic interviews, 93 participants (43.1\%) were classified as the addictive group and $123(56.9 \%)$ as the nonaddictive group under clinical impression, respectively. No participant had the diagnoses listed in criteria $\mathrm{C}$ or participated in Internet activity involved mainly in gambling or sexual behaviors. Besides, 113 participants (86 in addictive and 27 in nonaddictive groups) were found to have any 1 characteristic of functional impairment due to Internet use according to criteria B of the DC-IA-C. Among them, 89 (78.8\%) had impaired academic performance, 55 $(48.7 \%)$ reduced or had given up important social and recreational activities because of Internet use, 4 (3.5\%) failed to fulfill family obligations, and no one had impairment of work performance or repeatedly legal problems. Furthermore, the diagnostic accuracy, Cohen $\kappa$ value, and DOR of criteria B were $84.7 \%, 0.70$, and 51.49 , respectively.

Evaluation for fitness of cutoff point of criteria A in DCIA-C is shown in Table 2 . Because there is 1 cell with zero value in cutoff point of $7 / 8$ and $8 / 9$, the DOR was unable to be calculated; thus, the best cutoff point was determined by diagnostic accuracy and Cohen $\kappa$ value. The result revealed that the $5 / 6$ cutoff point had the best diagnostic accuracy (95.9\%) and Cohen $\kappa$ value (0.91). Besides, it had high specificity (92.4\%), sensitivity (98.4\%), PPR (97.7\%), and NPR (94.6\%).
According to the DC-IA-C with the 5/6 cut off point, 87 (40.3\%) and $129(59.7 \%)$ participants were diagnosed as Internet addiction group and non-Internet addiction group. The receiver operating characteristics analysis for the CIAS based on the DC-IA-C revealed that the area under curve was $87.7 \%$, indicating that CIAS had good diagnostic efficiency for Internet addiction. The sensitivity, specificity, diagnostic accuracy, PPR, NPR, Cohen $\kappa$ value, and DOR of indicated cutoff points of the CIAS are shown in Table 3. The cutoff point of 67/68 was the best point to discriminate cases with Internet addiction from noncases, with a high diagnostic accuracy (81.5\%), Cohen $\kappa$ value (0.61), DOR (18.22), and specificity (92.6\%). Meanwhile, the cutoff point of 63/64 on the CIAS resulted in a high sensitivity $(83.9 \%)$ and NPR $(87.6 \%)$ and an acceptable specificity $(76.7 \%)$. These results showed that $63 / 64$ is an optimal cutoff point for screening of Internet addiction.

According to the DC-IA-C with the 5/6 cutoff point in criteria $\mathrm{A}$, demographic and Internet-use characteristics were compared between the case and noncase groups (Table 4). The results indicated that the subjects in the case group were more likely to be male $\left(\chi^{2}=11.34, P=.001\right)$, had a higher frequency of Internet use $\left(\chi^{2}=38.94, P<\right.$ $.001)$, spent more time on Internet use $\left(\chi^{2}=56.36, P<\right.$ $.001)$, and played online games $\left(\chi^{2}=40.68, P<.001\right)$ more than those in the noncase group.

\section{Discussion}

Although there is still debate about whether Internet addiction is a distinct disorder or a behavioral problem secondary to other disorders [26], it has become an important behavioral problem deserving further research in modern society. Until now, it had not been classified or defined in $D S M-I V-T R$. In this study, we proposed the DC-IA-C according to the DC-IA-A. Different from the criteria proposed by Shapira et al [5] or Aboujaoude et al [12], the cutoff point of criteria A in the DC-IA-C was determined by empirical study, and the sensitive and specificity could be provided. The diagnostic criteria for Internet addiction

Table 2

The fitness of criteria A and cutoff point of DC-IA-C for diagnosis of Internet addiction in college students based on clinical impression

\begin{tabular}{|c|c|c|c|c|c|c|c|}
\hline & Sensitivity (\%) & Specificity (\%) & PPR (\%) & NPR (\%) & $\mathrm{DA}^{\mathrm{a}}(\%)$ & $\kappa^{\mathrm{b}}$ & DOR \\
\hline Functional impairment & 93.5 & 78.2 & 76.1 & 94.2 & 84.7 & 0.70 & 51.49 \\
\hline 0/1 cutoff point & 93.5 & 79.0 & 76.8 & 94.2 & 85.2 & 0.71 & 54.03 \\
\hline $1 / 2$ cutoff point & 93.5 & 80.6 & 78.2 & 94.3 & 86.1 & 0.72 & 59.72 \\
\hline $2 / 3$ cutoff point & 93.5 & 84.7 & 81.9 & 94.6 & 88.4 & 0.77 & 79.21 \\
\hline $3 / 4$ cutoff point & 93.5 & 87.1 & 84.3 & 94.7 & 89.8 & 0.80 & 96.75 \\
\hline $4 / 5$ cutoff point & 93.5 & 96.8 & 95.6 & 95.2 & 95.4 & 0.91 & 430 \\
\hline $5 / 6$ cutoff point & 92.4 & 98.4 & 97.7 & 94.6 & 95.9 & 0.91 & 740.71 \\
\hline $6 / 7$ cutoff point & 77.2 & 98.4 & 97.3 & 85.3 & 89.4 & 0.78 & 206.24 \\
\hline $7 / 8$ cutoff point & 48.9 & 100.0 & 100.0 & 72.5 & 78.2 & 0.52 & $\mathrm{X}$ \\
\hline $8 / 9$ cutoff point & 21.7 & 100.0 & 100.0 & 63.3 & 66.7 & 0.24 & $\mathrm{X}$ \\
\hline
\end{tabular}

\footnotetext{
${ }^{a}$ Diagnostic accuracy.
}

${ }^{b}$ Cohen $\kappa$. 
Table 3

The fitness of indicated cutoff points of the CIAS for diagnosis of Internet addiction in college students based on the DC-IA-C

\begin{tabular}{llllllll}
\hline $\begin{array}{l}\text { Cutoff } \\
\text { point }\end{array}$ & $\begin{array}{l}\text { Sensitivity } \\
(\%)\end{array}$ & $\begin{array}{l}\text { Specificity } \\
(\%)\end{array}$ & $\begin{array}{l}\text { PPR } \\
(\%)\end{array}$ & $\begin{array}{l}\text { NPR } \\
(\%)\end{array}$ & $\begin{array}{l}\mathrm{DA}^{\mathrm{a}} \\
(\%)\end{array}$ & $\kappa^{\mathrm{b}}$ & DOR \\
\hline $58 / 59$ & 90.8 & 61.2 & 61.2 & 90.8 & 73.2 & 0.48 & 15.60 \\
$59 / 60$ & 90.8 & 62.0 & 61.7 & 90.9 & 73.6 & 0.49 & 16.12 \\
$60 / 61$ & 87.4 & 67.4 & 64.4 & 88.8 & 75.5 & 0.52 & 14.31 \\
$61 / 62$ & 87.4 & 70.5 & 66.7 & 89.2 & 77.3 & 0.55 & 16.55 \\
$62 / 63$ & 86.2 & 72.1 & 67.6 & 88.6 & 77.8 & 0.56 & 16.15 \\
$63 / 64$ & 83.9 & 76.7 & 70.9 & 87.6 & 79.6 & 0.59 & 17.21 \\
$64 / 65$ & 82.8 & 78.3 & 72.0 & 87.1 & 80.1 & 0.60 & 17.31 \\
$65 / 66$ & 80.5 & 81.4 & 74.5 & 86.1 & 81.0 & 0.61 & 18.01 \\
$66 / 67$ & 74.7 & 82.9 & 74.7 & 82.9 & 79.6 & 0.58 & 14.37 \\
$67 / 68$ & 74.7 & 86.0 & 78.3 & 83.5 & 81.5 & 0.61 & 18.22 \\
$68 / 69$ & 65.5 & 88.4 & 79.2 & 79.2 & 79.2 & 0.55 & 14.44 \\
$69 / 70$ & 60.9 & 90.7 & 81.5 & 77.5 & 78.7 & 0.54 & 15.20 \\
\hline
\end{tabular}

a Diagnostic accuracy.

b Cohen $\kappa$.

constructed in this study can provide health care professionals with a means to communicate and make comparisons of clinical cases.

Functional impairment is required to make any psychiatric diagnosis according to DSM-IV-TR [11]. A previous study also raised the necessity of functional impairment for the diagnosis of Internet addiction [27]. We propose criterion B for making a diagnosis of Internet addiction to remind clinicians of the importance of evaluating functional impairment secondary to Internet use. Compared to the DC-IA-A, criteria B has been modified in DC-IA-C and revealed to have good discriminative ability for Internet addiction.

Because many college students had part-time work, the impairment of work performance should be included in the criteria. However, no college students had impaired work performance due to Internet use in this study. The characteristics of their work, which is mostly part-time, time-limited, and nonprofessional, may partly explain the result. Further studies are needed to examine whether this item has more importance for an adult group than for college students. No participant had legal problems because of Internet behavior in this study. However, the impaired function caused by several specific maladaptive Internet behaviors (eg, hacker, child pornography, and crime in online gaming) might be demonstrated with repeatedly legal problems according to clinical experience. Thus, we suggest "repeated legal problems" should be listed in criteria B. However, whether it is helpful for discriminating Internet addiction from other normal Internet use should be evaluated in further studies.

The diagnostic criterion A of the DC-IA-C include preoccupation, uncontrolled impulse, usage more than intended, tolerance, withdrawal, impairment of control, excessive time and effort spent on Internet use, and impairment of decision-making ability. Preoccupation and uncontrolled impulse resemble diagnostic criteria of patho- logical gambling in DSM-IV-TR [11]. The other 7 diagnostic criteria in criteria A are similar to the diagnostic criteria for substance dependence in DSM-IV-TR [11]. This result may indicate that Internet addiction had shared the core symptoms of substance dependence and impulse control disorder. However, there is no sufficient evidence to make any conclusion in classifying Internet addiction as an impulse control disorder as other behavioral addictions such as pathological gambling or shopping mania.

The results revealed that 6 criteria were required to fulfill criteria A to make the diagnosis of Internet addiction. The cutoff point seems to be higher than the DSM-IV-TR diagnostic criteria for substance dependence (3 or more of 7 criteria) and pathological gambling (5 or more of 10 criteria) [11]. It may be partially accounted for that Internet use is usually available in colleges and students can use the Internet for the purposes of studies and recreational activities. Therefore, more characteristic symptoms are required to differentiate between problematic and normal Internet use.

Because there is no participating case that fulfilled the excluding criteria in criteria $\mathrm{C}$, its application could not be tested in this article. Shaffer et al [26] have criticized what the object of Internet addiction is and suggest that other more primary disorders may provide better explanations for excessive computer use. Thus, excessive gambling or sexual

Table 4

Comparisons of the characteristics of college students with and without Internet addiction diagnosed by the DC-IA-C

\begin{tabular}{|c|c|c|c|}
\hline & \multicolumn{2}{|c|}{$\begin{array}{l}\text { Internet addiction } \\
\quad(\text { DC-IA-C) }\end{array}$} & \multirow[t]{2}{*}{$\chi^{2}$} \\
\hline & Yes, no. $(\%)$ & No, no. (\%) & \\
\hline \multicolumn{4}{|l|}{ Sex } \\
\hline Male & $65(74.7)$ & $67(51.9)$ & $11.34 * * *$ \\
\hline Female & $22(25.3)$ & $62(48.1)$ & \\
\hline \multicolumn{4}{|l|}{ Clinical impression } \\
\hline Case of Internet addiction & $85(97.7)$ & $7(5.4)$ & \\
\hline Non-case of Internet addiction & $2(2.3)$ & $122(94.6)$ & $180.94 * * *$ \\
\hline \multicolumn{4}{|l|}{$67 / 68$ cutoff point of CIAS } \\
\hline Case of Internet addiction & $65(74.7)$ & $18(14.0)$ & $81.07 * * *$ \\
\hline Non-case of Internet addiction & $22(25.3)$ & $111(86.0)$ & \\
\hline \multicolumn{4}{|l|}{$63 / 64$ cutoff point of CIAS } \\
\hline Case of Internet addiction & $73(83.9)$ & $30(23.3)$ & $76.62 * * *$ \\
\hline Non-case of Internet addiction & $14(16.1)$ & $99(76.7)$ & \\
\hline \multicolumn{4}{|l|}{ Time of Internet use per week } \\
\hline$>30 \mathrm{~h}$ & $51(58.6)$ & $14(10.9)$ & $56.36^{* * *}$ \\
\hline$\leq 30 \mathrm{~h}$ & $36(41.4)$ & $115(89.1)$ & \\
\hline \multicolumn{4}{|l|}{ Frequency of Internet use } \\
\hline $\begin{array}{l}\text { Intermediate (twice a week } \\
\text { or less) }\end{array}$ & $1(1.1)$ & $17(13.2)$ & $38.94 * * *$ \\
\hline Usually (3-5 times per week) & $1(1.1)$ & $34(26.4)$ & \\
\hline Nearly every day & $85(97.7)$ & $78(60.5)$ & \\
\hline \multicolumn{4}{|l|}{ Internet activity } \\
\hline Online gaming & $50(57.5)$ & $21(16.3)$ & $40.68 * * *$ \\
\hline Interpersonal activities & $20(23.0)$ & $68(52.7)$ & \\
\hline Others & $17(19.5)$ & $40(31.0)$ & \\
\hline
\end{tabular}


behavior online may be part of these excessive behaviors in the virtual or real world. However, characteristics of Internet use, such as access, affordability, and anonymity as outlined by Cooper [28], would make the object of addiction more attractive and contribute to the addiction potential.

Meanwhile, some cases are addictive to such objects only online but not in the real world in our clinical experience. Thus, their behavior might not be completely explained only by the primary object of addiction. Accordingly, we suggest that the object of Internet addiction should include (1) the specific behavior existing only in Internet use (eg, download); (2) the behavior online is totally different to that in real life (eg, online chatting and gaming); and (3) some behaviors, for example gambling, have addictive potentials only significantly enforced by Internet use. For the latter behaviors, if there is no difference between real or virtue addictive behavior, it is not necessary to make a diagnosis of Internet addiction. Thus, other behavior addictions that may be classified in impulse control disorder or paraphilia should be listed in excluding criteria to make further diagnosis. However, further research focusing on such types of Internet addiction is necessary to examine whether there is a difference between their addictive behavior in the Internet or in real life.

The determination for the cutoff point of the CIAS should take into account the epidemiological situation and the related consequences of false-negative and false-positive test results [20]. In the 2-stage approach, a higher sensitivity with accepted specificity at the first screening step is desirable. Following the principle, we suggest the cutoff point 63/64 of the CIAS to be a first-step screening tool with sensitivity of $83.9 \%$. On the other hand, optimizing diagnostic efficacy could be essential for the 1-stage investigation for community study. In this study, the diagnostic cutoff point $(67 / 68)$ constitutes the best point for the diagnostic accuracy (81.5\%), Cohen $\kappa$ value (0.61), and DOR (18.22). This discriminative potential made the CIAS appropriate for use as a reliable diagnostic tool in massive epidemiological surveys because it could provide the estimated prevalence and identification of the case group.

The results of the study also support the notion that the diagnostic criteria proposed in this study can discriminate between college students with different severities of Internet use. It was found that college students with Internet addiction were more likely to be male, which is congruent with a previous study [1]. It may be explained by the different social roles between males and females as a previous report has suggested [29]. This study also found that college students with Internet addiction were more likely to play online games. The feeling of control, synchronous interactive quality, the mask of identity, and the freedom of self-representation in online gaming [30] may provide higher incentive motivation than other Internet activities for college students.

The DC-IA-C providing descriptions for clinical symptoms could enable clinicians to diagnose if college students have Internet addiction and to intervene. Besides, use of DC-
IA-C may enhance agreement among clinicians and investigators. It could improve the communication and comparison of Internet addiction between health care professionals. Besides, it could also benefit further research to evaluate the course, prognosis, risk factors and mechanism of Internet addiction, which is essential for developing preventive and treatment schedule. On the other hand, the cutoff point of CIAS in this study could identify or screen college students with Internet addiction in a 1-stage or 2stage epidemiological investigation, respectively. It makes sizeable epidemiological research for Internet addiction possible. Besides, screening Internet addiction for every patient with an interview was inefficacious in the clinical setting. The CIAS with its screening cutoff point could become an appropriate screening instrument for Internet addiction in clinical settings.

This study has the following limitations. Firstly, because the participants were not recruited by random sampling, the rate of Internet addiction could not represent the prevalence in all college students. Secondly, the evaluation for Internet addiction relied solely on self-reported information in a diagnostic interview. Aside from observation and evaluation during the interview, more than one information source to investigate Internet addiction may provide clearer information. Thirdly, the 3-month monitoring duration suggested in the criteria had not been tested. Further studies to investigate the relapse potential and duration of Internet addiction are necessary to support the criteria.

\section{Conclusion}

This study established the DC-IA-C according to the analysis of an empirical community survey for college students. The DC-IA-C was composed of 3 main criteria, including characteristic symptoms of Internet addiction, functional impairment secondary to Internet use, and exclusive criteria. The DC-IA-C had high diagnostic accuracy and Cohen $\kappa$ value and can provide health care professionals with a means to identify college students with Internet addiction. Besides, the screening and diagnostic cutoff points of the CIAS developed in this study could provide a first-aid screening instrument in clinical practice or a discriminative instrument in massive survey for Internet addiction of college students.

\section{Acknowledgment}

This study was supported by a grant of the National Science Council of Taiwan (NSC 94-2314-B-037-099).

\section{References}

[1] Morahan-Martin J, Schumacher P. Incidence and correlates of pathological Internet use among college students. Comput Hum Behav 2000;16:13-29. 
[2] Scherer K. College life on-line: healthy and unhealthy Internet use. J Coll Stud Dev 1997;38:655-65.

[3] Young KS. Internet addiction: The emergence of a new clinical disorder. Cyberpsychol Behav 1998;1:237-44.

[4] Chou C, Condron L, Belland JC. A review of the research on Internet addiction. Educ Psychol Rev 2005;17:363-88.

[5] Shapira NA, Lessig MC, Goldsmith TD, Szabo ST, Lazoritz M, Gold MS, et al. Problematic Internet use: proposed classification and diagnostic criteria. Depress Anxiety 2003;17:207-16.

[6] Holden C. 'Behavioral' addictions: do they exist? Science 2001;294:980-2.

[7] Martin PR, Petry NM. Are non-substance-related addictions really addictions? Am J Addict 2005;14:1-3.

[8] Anderson KJ. Internet use among college students: an exploratory study. J Am Coll Health 2001;50:21-6.

[9] American Psychiatric Association. Diagnostic and statistical manual of mental disorders. 4th ed. Washington (DC): American Psychiatric Publishing, Inc.; 1994.

[10] Shapira NA, Goldsmith TD, Keck PE, Khosla UM, McElroy SL. Psychiatric features of individuals with problematic Internet use. J Affect Disord 2000;57:267-72.

[11] American Psychiatric Association. Diagnostic and statistical manual of mental disorders. 4th ed., Text Revision. Washington (DC): American Psychiatric Association; 2000.

[12] Aboujaoude E, Koran LM, Gamel N, Large MD, Serpe RT. Potential markers for problematic Internet use: a telephone survey of 2,513 adults. CNS Spectr 2006;11:750-5.

[13] Ko CH, Yen JY, Chen CC, Chen SH, Yen CF. Proposed diagnostic criteria of Internet addiction for adolescents. J Nerv Ment Dis 2005;193:728-33.

[14] Murphy JM. Symptom scales and diagnostic schedules in adult psychiatry. In: Tsuang MT, editor. Textbook in psychiatric epidemiology. 2nd ed. New York: Wiley-Liss, Inc.; 2002. p. 273-332.

[15] Chen SH, Weng LC, Su YJ, Wu HM, Yang PF. Development of Chinese Internet Addiction Scale and its psychometric study. Chin J Psychol 2003;45:279-94.

[16] Ko CH, Yen JY, Yen CF, Chen CC, Yen CN, Chen SH. Screening for Internet addiction: an empirical study on cut-off points for the Chen Internet Addiction Scale. Kaohsiung J Med Sci 2005;21:545-51.
[17] Yen JY, Yen CF, Chen CC, Chen SH, Ko CH. Family factors of Internet Addiction and substance use experience in Taiwanese adolescents. Cyberpsychol Behav 2007;10:323-9.

[18] Ko CH, Yen JY, Chen CC, Chen SH, Yen CF. Tridimensional personality of adolescents with Internet addiction and substance use experience. Can J Psychiatry 2006;51:887-94.

[19] Ko CH, Yen JY, Yen CF, Lin HC, Yang MJ. Factors predictive for incidence and remission of Internet addiction in young adolescents: a prospective study. Cyberpsychol Behav 2007;10:545-51.

[20] Greiner M, Pfeiffer D, Smith RD. Principles and practical application of the receiver-operating characteristic analysis for diagnostic tests. Prev Vet Med 2000;45:23-41.

[21] Glas AS, Lijmer JG, Prins MH, Bonsel GJ, Bossuyt PM. The diagnostic odds ratio: a single indicator of test performance. J Clin Epidemiol 2003;56:1129-35

[22] Hsu LM. Diagnostic validity statistics and the MCMI-III. Psychol Assess 2002;14:410-22.

[23] Almeida OP, Almeida SA. Short versions of the geriatric depression scale: a study of their validity for the diagnosis of a major depressive episode according to $I C D-10$ and $D S M-I V$. Int J Geriatr Psychiatry 1999;14:858-65.

[24] Lowe B, Spitzer RL, Grafe K, Kroenke K, Quenter A, Zipfel S, et al. Comparative validity of three screening questionnaires for DSM-IV depressive disorders and physicians' diagnoses. J Affect Disord 2004;78:131-40.

[25] Greiner M, Gardner IA. Epidemiologic issues in the validation of veterinary diagnostic tests. Prev Vet Med 2000;45:3-22.

[26] Shaffer HJ, Hall MN, Vander BJ. "Computer addiction": a critical consideration. Am J Orthopsychiatry 2000;70:162-8.

[27] Beard KW, Wolf EM. Modification in the proposed diagnostic criteria for Internet addiction. Cyberpsychol Behav 2001;4:377-83.

[28] Cooper A. Sexuality and the Internet: Surfing into the new millennium. Cyberpsychol Behav 1998;1:181-7.

[29] Ko CH, Yen JY, Chen CC, Chen SH, Yen CF. Gender differences and related factors affecting online gaming addiction among Taiwanese adolescents. J Nerv Ment Dis 2005;193:273-7.

[30] Leung L. Net-generation attributes and seductive properties of the Internet as predictors of online activities and Internet addiction. Cyberpsychol Behav 2004;7:333-48. 